\title{
Topological Plasmonic Chain with Retardation and Radiative Effects
}

\author{
Simon R. Pocock, ${ }^{*}{ }^{\dagger}$ Xiaofei Xiao, ${ }^{\dagger}$ Paloma A. Huidobro, ${ }^{\dagger}$ and Vincenzo Giannini ${ }^{\dagger, \ddagger, \S}$ \\ ${ }^{\dagger}$ Physics Department, Imperial College London, Blackett Laboratory, London SW7 2AZ, United Kingdom \\ ${ }^{\ddagger}$ Instituto de Estructura de la Materia (IEM-CSIC), Consejo Superior de Investigaciones Científicas, Serrano 121, 28006 Madrid, \\ Spain
}

\section{Supporting Information}

ABSTRACT: We study a one-dimensional plasmonic system with nontrivial topology: a chain of metallic nanoparticles with alternating spacing, which in the limit of small particles is the plasmonic analogue to the Su-Schrieffer-Heeger model. Unlike prior studies we take into account long-range hopping with retardation and radiative damping, which is necessary for the scales commonly used in plasmonics experiments. This leads to a non-Hermitian Hamiltonian with frequency dependence that is notably not a perturbation of the quasistatic model. We show that the resulting band structures are significantly different, but that topological features such as quantized Zak phase and protected edge modes persist because the system has the same eigenmodes as a chirally symmetric system. We discover the existence of retardation-induced topological phase transitions, which are not predicted in the SSH model. We find parameters that lead to protected edge modes and confirm that they are highly robust under disorder, opening up the possibility of protected hotspots at topological interfaces that could have novel applications in nanophotonics.

KEYWORDS: plasmonics, surface plasmons, topological insulator, edge states, hotspots, disorder, nanoparticle array

P lasmonic systems take advantage of subwavelength field confinement and the resulting enhancement to create hotspots, with applications in medical diagnostics, sensing and metamaterials. ${ }^{1,2}$ Arrays of metallic nanoparticles support surface plasmons that delocalize over the structure and whose properties can be manipulated by tuning the dimensions of the particles and their spacing. ${ }^{3-6}$ In particular, $1 \mathrm{D}$ and $2 \mathrm{D}$ arrays have significant uses in band-edge lasing ${ }^{7,8}$ and can be made to strongly interact with emitters. 9,10 Configurations of nanoparticle dimers have been shown to exhibit interesting physical properties; ${ }^{11}$ in the following we consider a nanoparticle dimer array in the context of topological photonics.

The rise of topological insulators, materials with an insulating bulk and conducting surface states that are protected from disorder, has inspired the study of analogous photonic and plasmonic systems. ${ }^{12-23}$ Topological photonics shows exciting potential for unidirectional plasmonic waveguides, ${ }^{24}$ lasing, ${ }^{25}$ and field enhancing hotspots with robust topological protection, which could prove useful for nanoparticle arrays on flexible substrates. ${ }^{26}$ Plasmonic and photonic systems provide a powerful platform to examine topological insulators without the complication of interacting particles and with interesting additional properties like non-Hermiticity. ${ }^{27-32}$ The lack of Fermi level simplifies the excitation of states, and the tunability made available by the larger scale allows for the study of disorder and defects in greater depth than electronic systems. ${ }^{33-35}$ They also simplify the study of topology in finite systems. ${ }^{36}$
One of the simplest topologically nontrivial models is that of $\mathrm{Su}$, Schrieffer, and Heeger ( $\mathrm{SSH}),{ }^{37,38}$ which features a chain of atoms with staggered hopping. There are analogous photonic systems such as zizag chains ${ }^{39,40}$ and the one-dimensional chain of metallic nanoparticles with alternating spacing (see Figure 1a). This chain has been studied in the quasistatic (QS) limit, where the dimensions of the chain are much less than the wavelength, $k d \ll 1 .^{41-43}$ In fact, when damping is neglected and only nearest neighbors considered the one-dimensional chain and the SSH model are physically equivalent. However, the QS limit has been shown to be insufficient for describing the band structures of equally spaced chains of nanoparticles ${ }^{44-48}$ whose radius and spacing are in the commonly studied regimes for plasmonic metamaterials. ${ }^{4-51}$ Retardation and radiative losses become increasingly significant for larger particles, so these effects must be treated properly if we are to understand the physics at experimentally realistic scales. Recently, Downing and Weick took this into account using an open quantum system approach. ${ }^{52}$

In the following work we present a treatment of the staggered one-dimensional plasmonic chain that takes into account retardation and radiative effects over long-range, generating a natural $1 \mathrm{D}$ topological insulator model that complements those already in the literature. ${ }^{53-56}$ The model breaks chiral symmetry only trivially by adding an identity term

Received: January 29, 2018

Published: April 30, 2018 


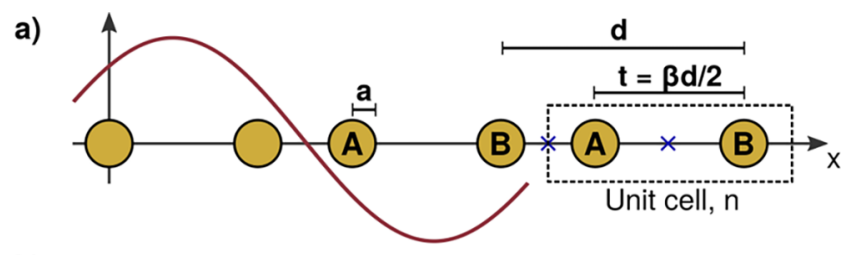

b)

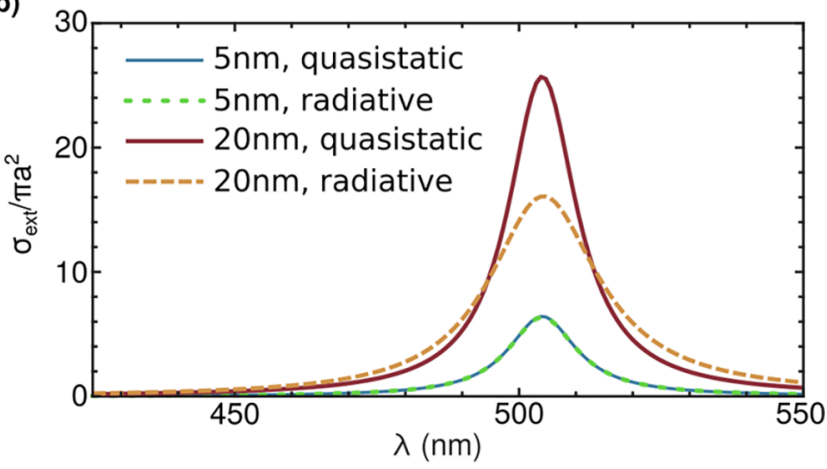

c)

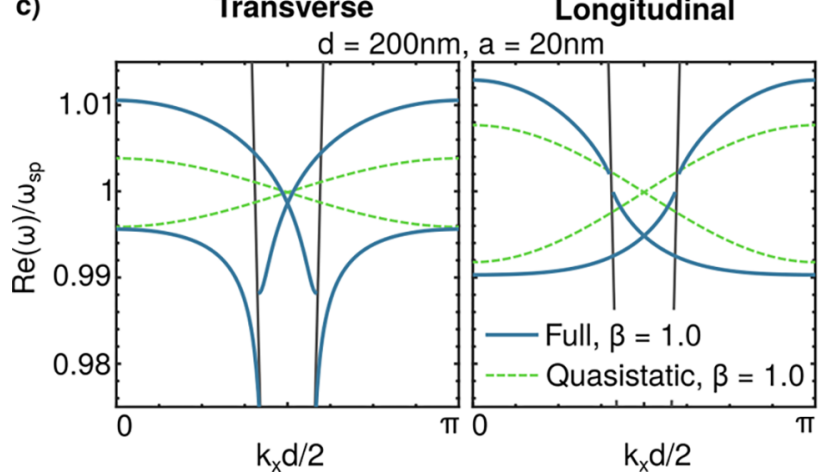

Figure 1. (a) Diagram of the topological plasmonic chain, with inversion centers of the chain marked by blue crosses. (b) Extinction cross section of a single gold nanoparticle with radius 5 and $20 \mathrm{~nm}$ embedded in glass, $\epsilon_{\mathrm{B}}=2.25$, comparing the effects of the QS polarizability against the radiative loss corrected polarizability. (c) Dispersion relations for an equally spaced chain, $\beta=1$, of nanoparticles, comparing the results of the nearest neighbor QS approximation (green dashed) and treating the full Green's function (blue solid), with light lines (black). $\omega$ is normalized to the surface plasmon resonance, $\omega_{\text {sp }}$.

to the Bloch Hamiltonian, which is non-Hermitian and has frequency dependence. We calculate band structures and compare to the QS approximation, showing that the system is indeed still topologically nontrivial because it shares eigenvectors with a chiral system. The transverse and longitudinal modes are shown to have notably different band structures, and in the transverse case it is shown that the Zak phase is not always the same as predicted by the QS approximation. In addition, we compare two methods of calculating the Zak phase and further confirm that for inversion symmetric systems it is possible to apply Zak's original results even in the case of non-Hermiticity. We go on to study the effects of disorder on the topologically protected edge states, which we find to be extremely robust.

\section{TOPOLOGICAL PLASMONIC CHAIN}

The plasmonic analogue of the SSH model is a chain of metallic nanoparticles with alternating spacing, as in Figure 1a. Particles have radius $a$ and unit cells length $d$, with the spacing between the $A$ and $B$ sublattices given by $t=\beta d / 2$, where $\beta$ acts as a tuning parameter. If the spacing of the particles is large enough compared to the radius of the spheres $(t, d-t \geq 3 a)$ the nanospheres can be treated as dipoles ${ }^{57}$ with dipole moments $\mathbf{p}_{n}$, and the system is described by the coupled dipole equations:

$$
\frac{1}{\alpha(\omega)} \mathbf{p}_{n}=\sum_{j \neq n} G\left(\mathbf{r}_{n j}, \omega\right) \mathbf{p}_{j}
$$

where $\mathrm{G}\left(\mathbf{r}_{n j}, \omega\right)$ is the free space $3 \times 3$ Green's dyadic, which depends on the separation of the dipoles, $\mathbf{r}_{n j}=\mathbf{r}_{n}-\mathbf{r}_{j}$ and complex frequency $\omega$.

The properties of the individual nanospheres are represented by the polarizability $\alpha(\omega)$, which in the quasistatic approximation is given by

$$
\alpha_{\mathrm{QS}}(\omega)=4 \pi a^{3} \epsilon_{0} \frac{\epsilon(\omega)-\epsilon_{\mathrm{B}}}{\epsilon(\omega)+2 \epsilon_{\mathrm{B}}}
$$

where $\epsilon(\omega)$ is the dielectric function of the metal, $\epsilon_{0}$ is the permittivity of free space, and $\epsilon_{\mathrm{B}}$ is the permittivity of the background dielectric. This neglects radiative damping, which is essential for the model to be consistent with the optical theorem. ${ }^{58}$ This is addressed by the radiative correction,

$$
\alpha(\omega)=\frac{\alpha_{Q S}(\omega)}{1-i \frac{k^{3}}{6 \pi \epsilon_{0}} \alpha_{Q S}(\omega)}
$$

where $k=\sqrt{\epsilon_{\mathrm{B}}} \omega / c=\sqrt{k_{x}^{2}+k_{y}^{2}+k_{z}^{2}}$ is the magnitude of the wavevector. Throughout this work we consider gold nanospheres using the Drude model,

$$
\epsilon(\omega)=\epsilon_{\infty}-\frac{\omega_{\mathrm{P}}^{2}}{\omega^{2}+i \omega / \tau}
$$

with $\epsilon_{\infty}=9.1, \omega_{\mathrm{P}}=1.38 \times 10^{16} \mathrm{rad} / \mathrm{s}$ and $1 / \tau=1.08 \times 10^{14}$ $\mathrm{rad} / \mathrm{s}$, ${ }^{59}$ embedded in a material like glass with the dielectric constant $\epsilon_{\mathrm{B}}=2.25$. We deliberately exclude interband transitions to make the effects of retardation more clear in this study, although we do not expect this to affect the existence of modes, ${ }^{17,41}$ as shown in the Supporting Information (SI). We also do not include a substrate, which could modify the dispersion relation of the plasmons, ${ }^{60,61}$ but is avoidable by making use of a transparent substrate and index matching with the surrounding material. ${ }^{10}$ Figure $1 \mathrm{~b}$ shows the normalized extinction cross section of a single nanoparticle, where the solid lines are quasistatic and the dashed make use of the radiative correction. For particles of $5 \mathrm{~nm}$ radius, the QS approximation agrees with the radiative correction, but for particles with radius $20 \mathrm{~nm}$ radiative losses strongly affect the extinction cross section by reducing and broadening the resonance over wavelength. Particles with radii above $5 \mathrm{~nm}$ are well described classically and do not require quantum size effects to be taken into account. ${ }^{62}$

The chain is confined to the $x$-axis, so the longitudinal $(x)$ and transverse $(y, z)$ parts of eq 1 decouple,

$$
\frac{1}{\alpha(\omega)} p_{\nu, n}=\sum_{j \neq n} G_{\nu}\left(r_{n j}, \omega\right) p_{\nu, j}
$$

where $\nu=x, y, z$ labels the orientation of the dipoles, and the hopping between dipoles is scalar,

$$
G_{x}\left(r_{n j}, \omega\right)=\frac{2 e^{i k r_{n j}}}{4 \pi \epsilon_{0} r_{n j}^{3}}\left[1-i k r_{n j}\right]
$$




$$
G_{y, z}\left(r_{n j}, \omega\right)=\frac{-e^{i k r_{n j}}}{4 \pi \epsilon_{0} r_{n j}^{3}}\left[1-i k r_{n j}-k^{2} r_{n j}^{2}\right]
$$

with $\omega$ dependence contained within $k$.

Similar theory has been used in many studies of equally spaced chains. ${ }^{63-71}$ However, unlike this work, previous studies of the staggered chain have ignored Drude damping $(1 / \tau=0)$ and taken the QS approximation with nearest neighbor hopping to solve the system. ${ }^{41-43}$ This neglects retardation by assuming that the dimensions of the chain are very small compared to the wavelength $k d \ll 1$. In this limit,

$$
G_{\nu}^{\mathrm{QS}}\left(r_{n j}\right)=m_{\nu} \frac{1}{4 \pi \epsilon_{0} r_{n j}^{3}}
$$

where $m_{\nu}=2$ for the longitudinal case and -1 for transverse. This removes all $\omega$ dependence from $G_{\nu}$ and neglects the intermediate and long-range dipolar interactions. The resulting nearest neighbor, real, staggered hopping provides a close analogue to the SSH model, apart from a transformation from the eigenvalues to the frequency $\omega$.

For gold nanoparticles embedded in glass the nonradiative surface plasmon resonance $\omega_{\mathrm{sp}}=\omega_{\mathrm{P}} / \sqrt{\epsilon_{\infty}+2 \epsilon_{\mathrm{B}}}$ corresponds to the wavelength $\lambda_{\mathrm{sp}}=504 \mathrm{~nm}$. Figure 1c shows the dramatic difference between the QS approximation and the retarded treatment for an evenly spaced chain $(\beta=1)$ when $d=200 \mathrm{~nm}$, on the same order as $\lambda_{\mathrm{sp}}$. Green dashed lines show bands resulting from the QS approximation, which are therefore symmetric around $\omega_{\mathrm{sp}}$. The blue solid lines show the result of including retardation and radiative effects. As has previously been shown for the evenly spaced chain, retardation leads to polariton splitting and discontinuities at the light lines $k=$ $\pm k_{x}{ }^{44,46}$ which are completely absent in the QS approximation. The difference is even greater for the transverse polarization due to the extremely long-range $\sim \exp (i k r) / r$ term in the full dipolar interactions. When including retardation and radiative losses hopping becomes complex and long-range, giving rise to a non-Hermitian topologically nontrivial Hamiltonian.

Bulk Bloch Hamiltonian. Topologically nontrivial systems exhibit a bulk-boundary correspondence, where properties of the bulk, here the Zak phase, ${ }^{72}$ predict the existence or absence of edge states in the finite case. ${ }^{73}$ We study the bulk by way of an infinite chain, where we relabel the two particles in the unit cell $A$ and $B$ as in Figure 1a, apply Bloch's theorem, and arrive at the equations

$$
\mathcal{G}_{\nu}\left(k_{x}, \omega\right)\left(\begin{array}{l}
p_{\nu}^{A} \\
p_{\nu}^{B}
\end{array}\right)=\frac{1}{\alpha(\omega)}\left(\begin{array}{l}
p_{\nu}^{A} \\
p_{\nu}^{B}
\end{array}\right)
$$

where $\mathcal{G}_{\nu}\left(k_{x}, \omega\right)$ acts as an $\omega$-dependent non-Hermitian Bloch Hamiltonian that is, in matrix form,

$$
\begin{aligned}
& \mathcal{G}_{\nu}\left(k_{x}, \omega\right)= \\
& \left(\begin{array}{cc}
\sum_{n \in \mathbb{Z}}{ }^{\prime} G_{\nu}(n d, \omega) e^{i k_{x} n d} & \sum_{n \in \mathbb{Z}} G_{\nu}(n d+t, \omega) e^{i k_{x} n d} \\
\sum_{n \in \mathbb{Z}} G_{\nu}(n d-t, \omega) e^{i k_{x} n d} & \sum_{n \in \mathbb{Z}}{ }^{\prime} G_{\nu}(n d, \omega) e^{i k_{x} n d}
\end{array}\right)
\end{aligned}
$$

where the primed sum notation indicates that the sum does not include the $n=0$ term. The eigenvalues of $\mathcal{G}_{\nu}$ are $1 / \alpha(\omega)$, but the band structure is given by $\omega$. Topological properties of the system are associated with the eigenvectors $\mathbf{p}_{\nu}$.
As is the case for any two band Hamiltonian, it is possible to write $\mathcal{G}$ in terms of the Pauli matrices $\left\{\sigma_{i}\right\}$,

$$
\mathcal{G}\left(k_{x}\right)=g_{0}\left(k_{x}\right) I+\mathbf{g}\left(k_{x}\right) \cdot \boldsymbol{\sigma}
$$

with $g_{0}$ and $\mathbf{g}=\left(g_{x}, g_{y}, g_{z}\right)$ given by examining $\mathcal{G}_{\nu}$. The QS nearest neighbor approximation has strict chiral symmetry, also called sublattice symmetry, because there is no hopping from sites $A$ to $A$ or from $B$ to $B$. This can be expressed by the equation $\sigma_{z} H \hat{\sigma}_{z}=-\hat{H}$, which is true when $g_{0}=0=g_{z}$. For the retarded treatment, we still have $g_{z}=0$, but $g_{0} \neq 0$, which we will call "trivial" chiral symmetry breaking. Strict chiral symmetry leads to eigenvalues $1 / \alpha(\omega)$ that are symmetric around 0 , but in this trivially broken case they are symmetric around $g_{0}\left(k_{x}, \omega\right)$. This system still has inversion symmetry in the $x$-direction expressed by $\sigma_{x} \mathcal{G}_{\nu}\left(k_{x}\right) \sigma_{x}=\mathcal{G}_{\nu}\left(-k_{x}\right)$, with inversion centers marked by crosses in Figure 1a. This guarantees that the band structure is symmetric in $k_{x}$.

Calculating the band structure is a matter of fixing real $k_{x}$ and finding corresponding complex $\omega$ numerically, to solve

$$
\operatorname{det}\left(\mathcal{G}_{\nu}-\frac{1}{\alpha(\omega)} I\right)=0
$$

We discuss the implications of complex frequencies later. The calculation is complicated by the fact that the elements of $\mathcal{G}_{\nu}$ are infinite, slowly converging sums. Faster evaluation can be achieved by writing the sums in terms of polylogarithms and the Lerch transcendent, detailed in the SI. These analytical expressions show that, when $\beta=1$, the off-diagonals of $\mathcal{G}_{\nu}$ are zero at the edge of the Brillouin zone (BZ), at $k_{x} d / 2=\pi / 2$. Therefore, the eigenvalues are degenerate here, leading to a band crossing as in Figure 1c. This signifies a topological phase transition at $\beta=1$.

Figure 2 shows numerically calculated band structures for various choices of chain parameters $d$ and $a$, displaying only the real part of $\omega$. These results are supported by full Maxwell's equations simulations in the SI. Blue dashed lines show the $\beta=$ 1 case, and red solid lines show the $\beta=0.9$ (identical to $\beta=$ 1.1) case. For $\beta \neq 1$, a complex valued gap opens at the edge of the $\mathrm{BZ}$, which increases in magnitude with increasing $|\beta-1|$.

For small chain geometry $(d=50 \mathrm{~nm}, a=5 \mathrm{~nm})$ in Figure 2(a) the band structure is well approximated by the QS model (yellow line), which makes a reasonable prediction of the band gap but fails to predict the small deviations of the band structure at the light line in the transverse case. Already some asymmetry in $\operatorname{Re}(\omega)$ exists due to the trivial breaking of chiral symmetry.

Figure $2 \mathrm{~b}, \mathrm{c}$ demonstrates band structures with larger particles and spacing with $d \sim \lambda_{s p} / \sqrt{\epsilon_{\mathrm{B}}}$, well away from the QS limit. Once again the polariton splitting and $\operatorname{Re}(\omega)$ asymmetry are present, as well as discontinuities at the light line, as in the upper band for (b) longitudinal. The QS approximation, not shown for clarity, is poor here and completely fails to predict that a gap in $\operatorname{Re}(\omega)$ does not always open, such as in (b) transverse and (c) longitudinal. It is important to note that in these cases there still exists an gap in $\operatorname{Im}(\omega)$, such that there is no band crossing and associated topological phase transition, as discussed in the Complex Bands section. This means these cases can still be topological or trivial.

Zak Phase. The relevant topological number is the Zak phase, which for an Hermitian system like the SSH model is given by 

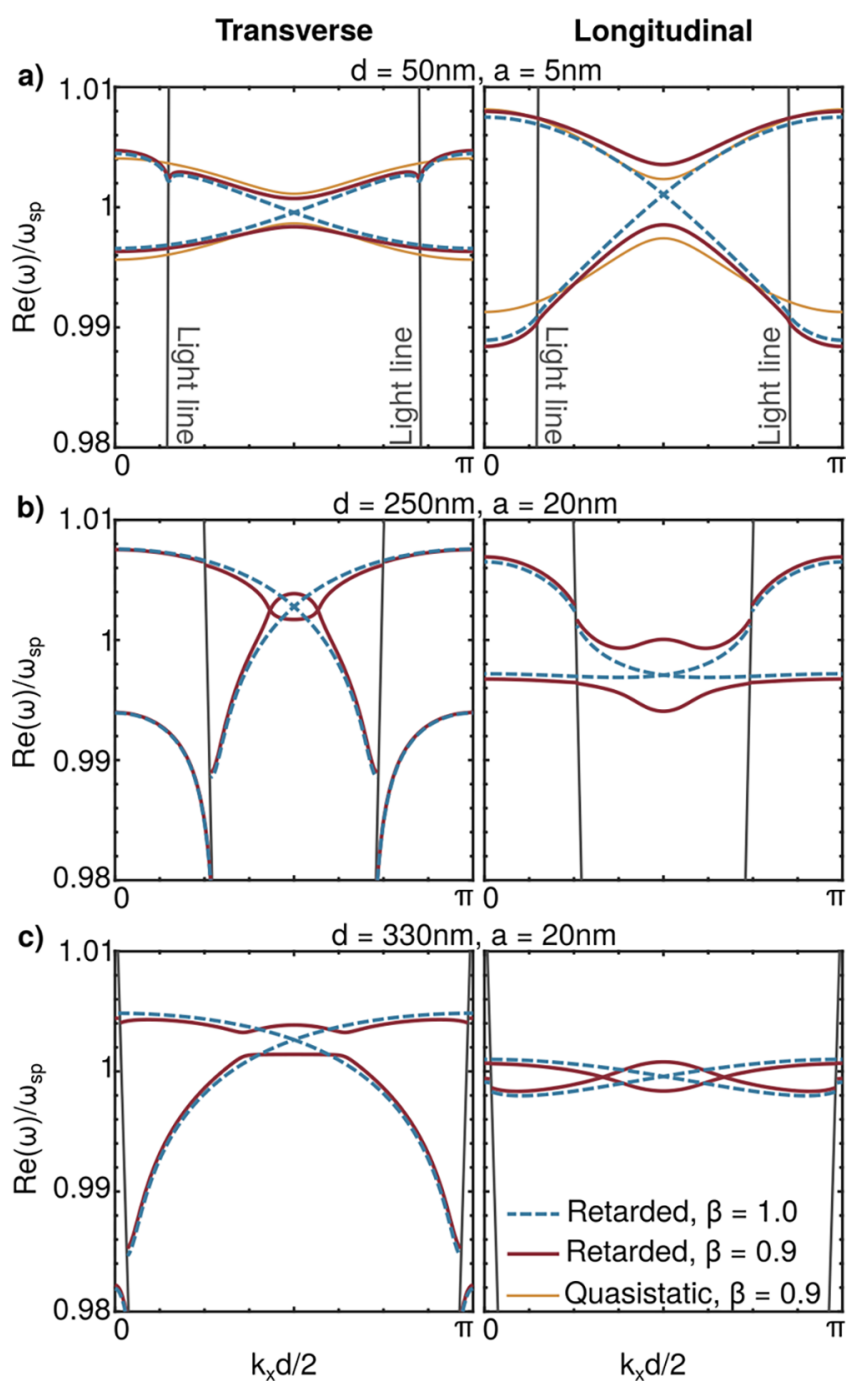

Figure 2. Band structures for the topological plasmonic chain for various choices of geometric parameters $d$ and $a$, with $\beta=0.9$ or 1.1. (a) Comparison of QS and retarded band structures near the QS regime. (b) and (c) show band structures further away from the QS regime and do not feature the yellow QS bands, which are similar to in (a).

$$
\gamma_{\mathrm{H}}=i \int_{-\pi / d}^{\pi / d} \mathbf{p}^{\dagger} \frac{\partial \mathbf{p}}{\partial k_{x}} \mathrm{~d} k_{x}
$$

However, the Hamiltonian $\mathcal{G}_{\nu}$ is non-Hermitian, so we must be more careful. The generalization of the Berry phase for nonHermitian systems, ${ }^{27}$ written in $1 \mathrm{D}$ for our system, is

$$
\gamma=i \int_{-\pi / d}^{\pi / d} \mathbf{p}_{\mathrm{L}}^{\dagger} \frac{\partial \mathbf{p}_{\mathrm{R}}}{\partial k_{x}} \mathrm{~d} k_{x}
$$

where $\mathbf{p}_{\mathrm{R}}$ and $\mathbf{p}_{\mathrm{L}}$ are normalized biorthogonal right and left eigenvectors, solving eq 9 and its Hermitian conjugate, respectively. It has been shown that chiral symmetry quantizes this non-Hermitian Zak phase. ${ }^{32}$ As discussed previously, our Hamiltonian breaks chiral symmetry trivially due to an additional identity term $g_{0}$. Since all vectors are eigenvectors of the identity, the system shares eigenvectors with a chirally symmetric counterpart (see SI) and the chiral symmetry result quantizing the Zak phase applies for this system too. In fact, the inversion symmetry of the system leads to quantization of the
Hermitian Zak phase as well, ${ }^{74}$ so that in this case both calculations have the same result,

$$
\gamma=\frac{\phi(\pi / d)-\phi(-\pi / d)}{2}
$$

where $\phi$ is the relative phase difference between $p^{\mathrm{A}}$ and $p^{\mathrm{B}}$. It follows that $\gamma$ is either 0 or $\pi$ modulo $2 \pi$. Since topological systems with chiral symmetry but no inversion symmetry can exist $^{32}$ the topological nature here arises because the system shares eigenvectors with a chirally symmetric system. When the Zak phase is $\gamma=\pi$, we expect topologically protected edge modes.

Figure 3a shows how $\phi\left(k_{x}\right)$ changes across half the Brillouin zone for the lower bands of Figure $2 b$. We only need half the
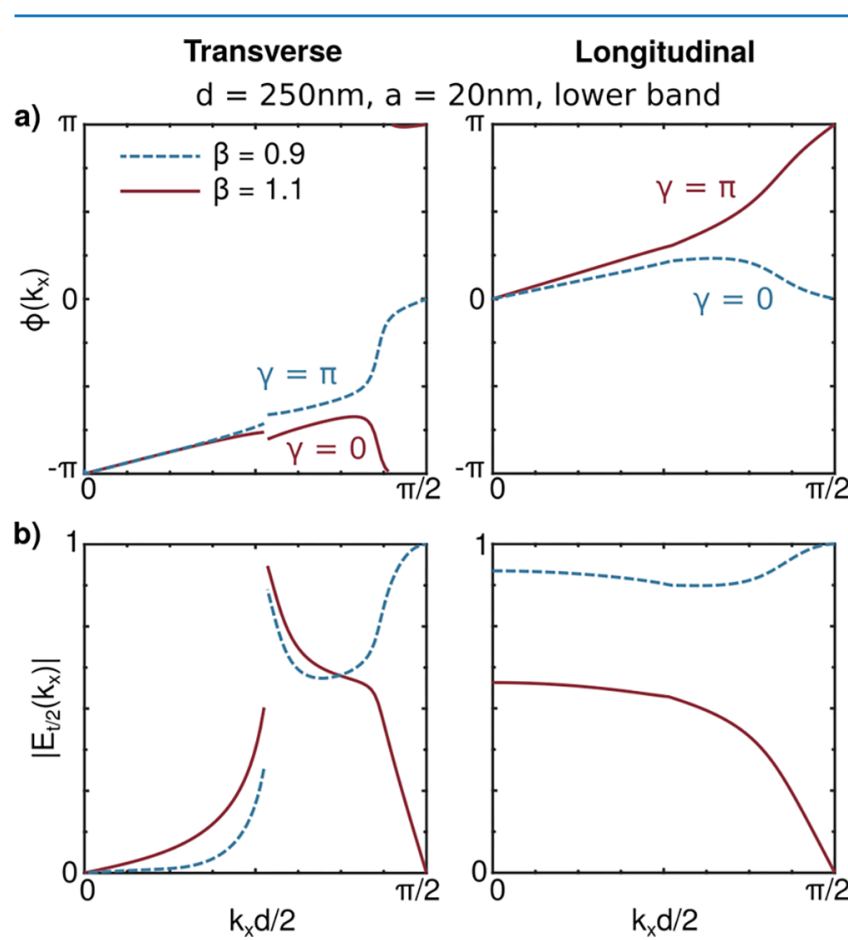

Figure 3. Representations of the calculation of the Zak phase $\gamma$, considering the lower band from Figure 2b. (a) Change of $\phi$ across the BZ modulo $2 \pi$. (b) Normalized electric field at the $k_{x}=t / 2$ inversion center. The upper band has the same topological number as the lower band in all examined cases.

BZ because inversion symmetry gives us the other half, with $\phi\left(-k_{x}\right)=-\phi\left(k_{x}\right)$. We examine $\beta$ either side of the topological phase transition at $\beta=1$. The longitudinal case has the same property as the SSH model and QS approximation, that $\gamma=\pi$ when $\beta>1$ and $\gamma=0$ when $\beta<1{ }^{41}$ Surprisingly, the transverse case is in the opposite topological phase to the longitudinal case for the same choice of unit cell when it has the same $\beta$. Of the example band structures given in Figure 2, all bands have the same topological properties as predicted by the SSH model, except (b) transverse. This implies that a topological phase transition occurs for the transverse polarized modes between (a) and (b) and again between (b) and (c).

We confirm these results by considering an alternative Zak phase calculation. Xiao et al. showed that, in photonic systems, the Zak phase is also given by the behavior of the electric field at the inversion centers $x=t / 2,(d+t) / 2$ of the chain, at the center $\left(k_{x}=0\right)$ and edge $\left(k_{x}=\pi / d\right)$ of the Brillouin zone (BZ). ${ }^{75}$ Considering the inversion center at $x=t / 2$, if $\mid E_{t / 2}\left(k_{x}=\right.$ 
$0) \mid$ and $\left|E_{t / 2}\left(k_{x}=\pi / d\right)\right|$ are both either zero or nonzero, we have $\gamma=0$. If $\left|E_{t / 2}\left(k_{x}=0\right)\right|$ and $\left|E_{t / 2}\left(k_{x}=\pi / d\right)\right|$ are opposite (one is zero while the other is nonzero), the Zak phase is given by $\gamma=$ $\pi$. The normalized magnitude of the electric field at $x=t / 2$ is shown across the $\mathrm{BZ}$ in Figure $3 \mathrm{~b}$, which agrees with the calculations of Figure 3a. If we take the opposite inversion center the Zak phases are switched. This method of determining the topological nature of the system relies on Zak's results ${ }^{72}$ originally for Hermitian systems, but inversion symmetry assures that any results for the Hermitian Zak phase is identical here to the non-Hermitian Zak phase.

Through the treatment of retardation, we have found an interesting phase transition for transverse polarized modes that was overlooked in previous studies of the topological plasmonic chain. We examine one of these phase transitions in Figure 4,

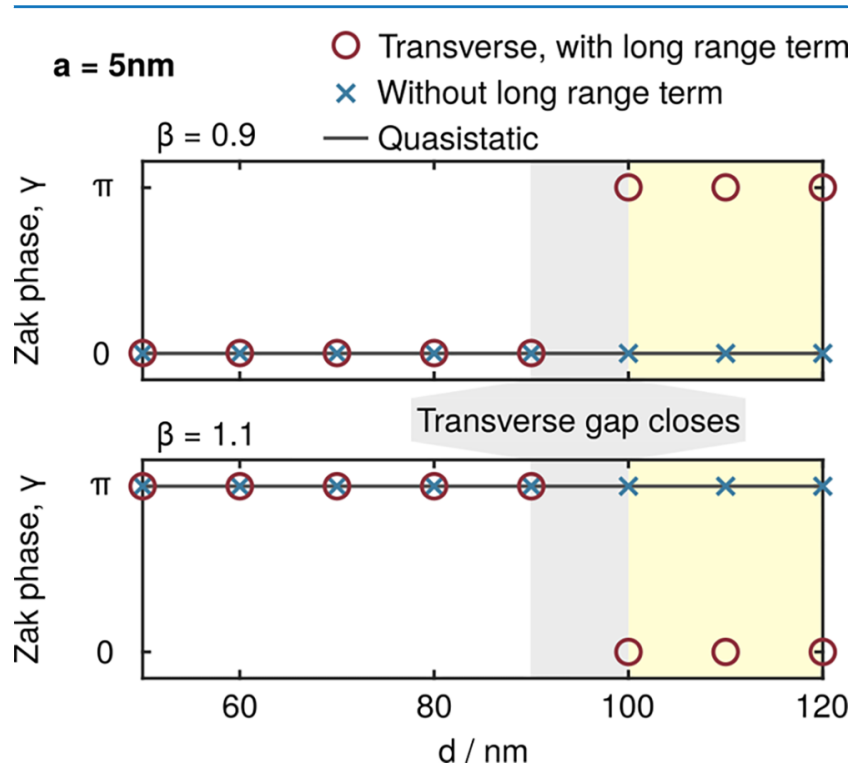

Figure 4. Retardation-induced Zak phase changes with particle spacing $d$, where the ratio $\beta$ is fixed. The transverse gap closes somewhere in the gray region and a phase transition occurs that is not predicted by the QS nearest neighbor approach. Red circles are the calculation with the full transverse Green's function in eq 7. Blue crosses show the case where the third long-range term is not included, equivalent to the longitudinal polarized modes.

where the Zak phases for chains with fixed radius $a=5 \mathrm{~nm}$ and $\beta=0.9,1.1$ are plotted with changing unit cell spacing, $d$. The Zak phase predicted by the QS model is shown by the horizontal dark gray line. Results obtained for transverse modes are given by red circles, which agree with the QS prediction for chain periods near the QS limit, then undergo a phase transition somewhere between $d=90$ and $100 \mathrm{~nm}$ in the gray region. Consequently, it enters the phase opposite to the QS prediction in the yellow region.

Topological phase changes occur when the product of the off diagonal elements of the matrix $\mathcal{G}_{\nu}\left(k_{x}, \omega\right)$ in eq 10 is zero, causing the gap to close. Changing the radius of the particles $a$ only impacts the eigenvalue $1 / \alpha(\omega)$, which could change where the gap closes in $d$ but not the existence of the gap closure. In fact, the zeros can only happen for the transverse case due to the third, long-range, term in the Green's function in eq 7. We study this by considering the Zak phase for a system which does not include this long-range term, given by the blue crosses in Figure 4. This case is equivalent to the longitudinal case, which has the same Green's function apart from a factor of -2 , which makes no difference to the closing of the gap. Here no phase change occurs with changing $d$, as predicted by the QS model, because the missing long-range term is responsible for the phase transition. Finally, we observe that the off diagonals of $\mathcal{G}_{\nu}\left(k_{x}, \omega\right)$ are transformed into each other by mapping $\beta \mapsto 2$ $-\beta$. If the gap closes for $\beta$ it will also close for $2-\beta$, so that phase transitions occur for $\beta$ symmetric around $\beta=1$, and are opposite due to the phase transition at $\beta=1$.

In the transverse case we have shown that when joining suitably long chains with the same $d$ and $a$, and with $\beta$ symmetric on either side of the topological phase transition $\beta=$ 1 , we still expect a topologically nontrivial interface featuring a topological edge mode. The longitudinal case is less restrictive due to the lack of retardation-induced phase transitions, as any chains with $\beta$ on opposite sides of $\beta=1$ have opposite Zak phase.

Finite Chains and Disorder. We now consider the implications of the topological phases in finite chains. Figure $5 a$ shows the eigenmodes of a finite chain with an example choice of parameters so that there is a gap in $\operatorname{Re}(\omega)$. The gap as defined by the bulk modes (blue) increases symmetrically away from $\beta=1$, where there is a topological phase transition. As expected, edge modes (yellow) appear in the gap when the Zak phase $\gamma=\pi$.

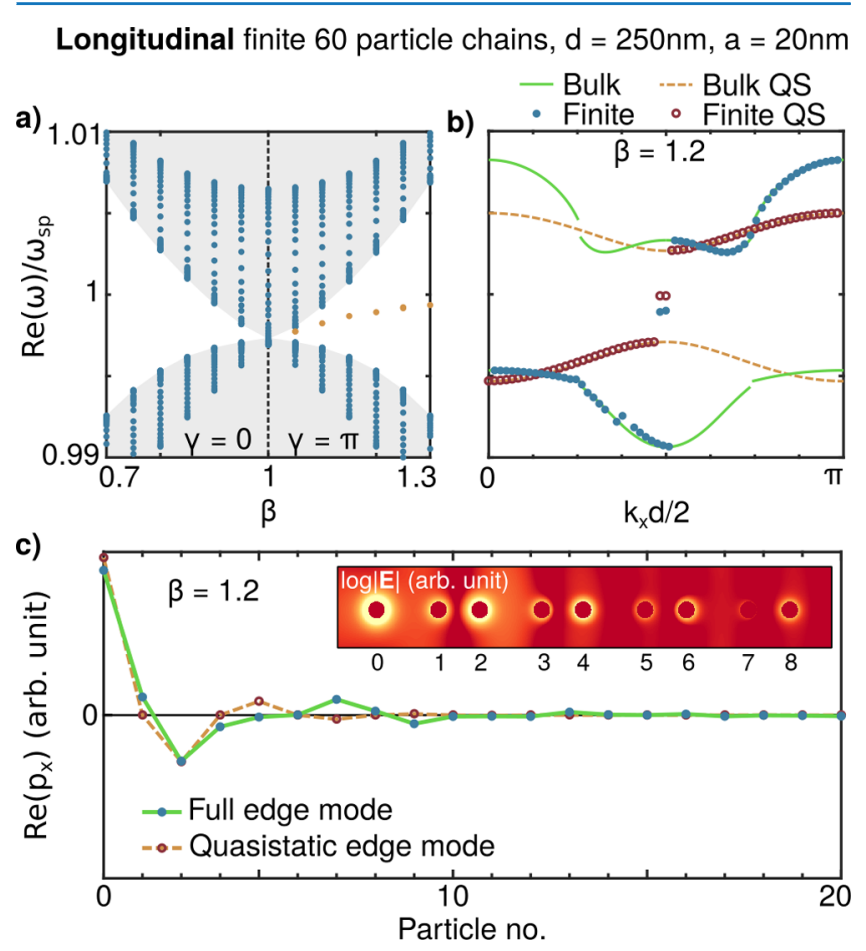

Figure 5. (a) Longitudinal eigenmodes of a finite 60 particle chain with varying $\beta$. As predicted by the Zak phase, edge states (yellow) exist when $\beta>1$. (b) Comparison of the quasistatic and retarded finite chain band structure for a choice of $\beta=1.2$, showing edge modes in the gap. (c) The real parts of the QS and retarded edge mode profiles of the leftmost end of the chain. Modes in the gap are symmetric and antisymmetric combinations of these mode profiles. Inset: $\log |\mathbf{E}|$ field outside of the particles for retarded left edge mode, excited by an evanescent plane wave perpendicular to the chain. Dark red corresponds to the weakest field, and bright yellow represents a stronger field. 
Bulk modes can be identified by their mode profiles, which are typically similar to normal modes of a chain, as in Figure $6 \mathrm{~b}$.

Longitudinal finite 60 particle chains, $d=250 \mathrm{~nm}, a=20 \mathrm{~nm}$
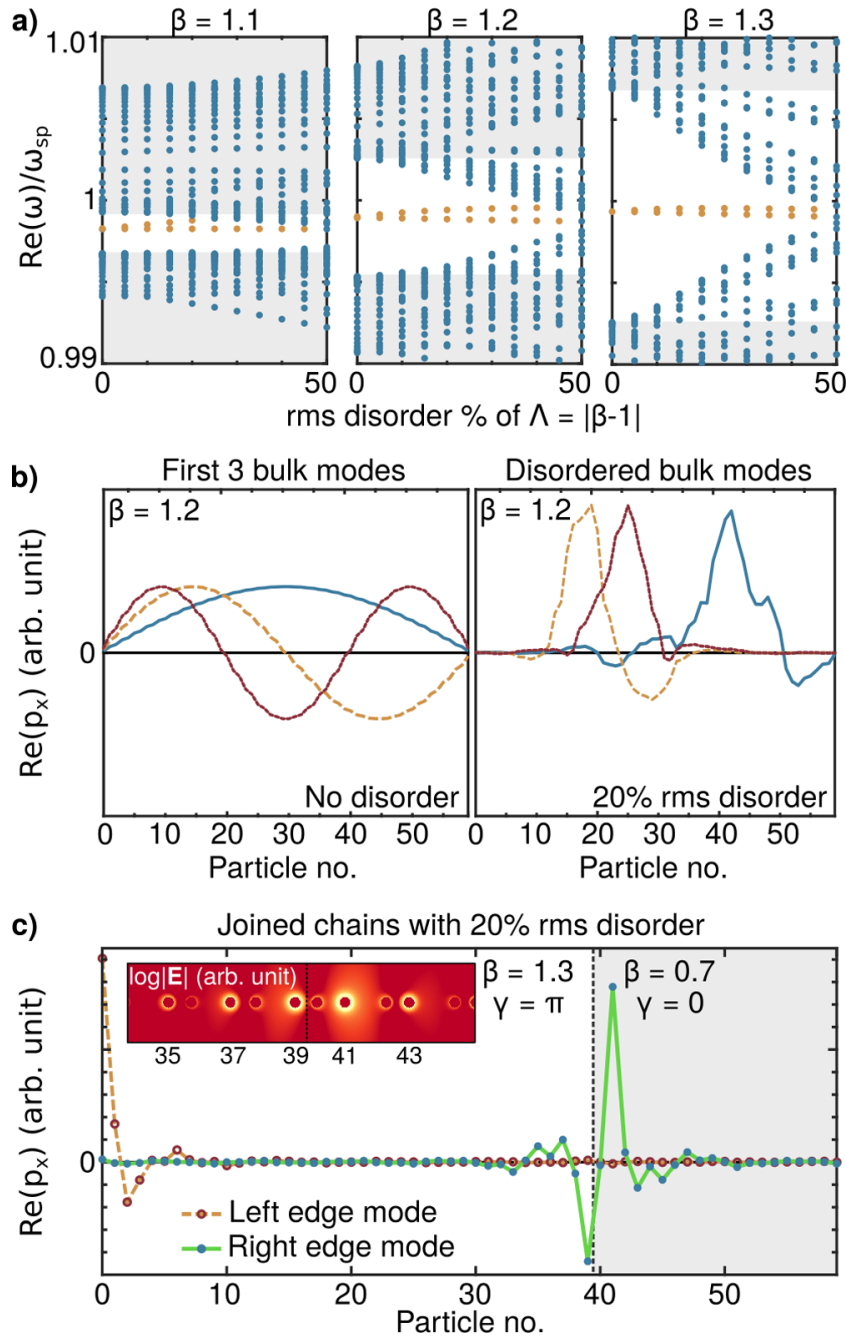

Figure 6. (a) Random disorder with the same seed is applied with increasing strength along the $x$ axis for three different choices of $\beta$. The original bulk is marked in gray, bulk modes are blue and edge modes are yellow. (b) Bulk modes of the of the system without disorder and with disorder, using the same seed as (a). (c) Joined chains with opposite $|\beta-1|$ values interfaced between particles 39 and 40 . Disorder uses a different seed to (a) and (b). Inset: $\log |\mathbf{E}|$ field outside of the particles, around the topological interface for the right edge mode excited by an evanescent plane wave perpendicular to the chain. Dark red corresponds to the weakest field, and bright yellow represents a stronger field.

They can therefore be ordered by assigning a mode number $n$, the number of times the sign of $\operatorname{Re}\left(p_{\nu}\right)$ changes plus 1 , and a $k_{x}$ given by

$$
k_{x} \frac{d}{2}=\frac{(N-2) n+1}{N(N-1)} \pi
$$

where $N$ is the number of particles in the chain, ${ }^{44}$ and $N=60$ in our calculations. These $\left(k_{x}, \omega\right)$ pairs are plotted for an example set of parameters in Figure $5 b$, where the finite QS approximation (red circles) and retarded system (blue dots) are compared. Bulk modes of the finite chain approximate the
Bloch bulk band structure in both cases, while the two edge modes exist instead in the gap.

Figure $5 \mathrm{c}$ shows the dipole moments of a set of particles near the edge, with a comparison between the QS edge mode (yellow dashed) and the retarded edge mode (green solid). The QS edge mode is fully supported on only the A sublattice due again to chiral symmetry, while the retarded edge mode exists on both sublattices. This is due to the long-range nature of the hopping, forcing the retarded case to be further from the fully dimerized limit than the QS case. This also explains why in the QS case the edge modes have energies fixed to $\operatorname{Re}(\omega)=\omega_{\text {sp }}$ but the retarded edge modes' energies are slightly different. The edge modes decay exponentially into the chain, although the retarded edge mode does so with a longer decay length. The real part of $p_{x}$ has a minimum at particle 5 before increasing and then decreasing again, because of the longer range, out of phase, dipole-dipole interactions, but the absolute value $\left|p_{x}\right|$ still decays monotonically on each sublattice into the chain as illustrated by the inset log $|\mathbf{E}|$ field.

When the gap has no real part, edge mode frequencies have an imaginary part so that they still sit in the imaginary valued gap for $\gamma=\pi$, which we discuss later, and have similar profiles to Figure $5 \mathrm{c}$. For the transverse case the extremely long-range dipole-dipole interactions $\sim \exp (i k r) / r$ appear to necessitate a very long chain in order to distinguish an edge mode, which is not accessible with our numerics. Therefore, we are restricted here to studying longitudinal edge modes on finite chains.

One of the most relevant properties that arises due to topology is the protection of the edge modes from disorder in the axis of the chain. In Figure 6 we apply disorder in the form of a random positive or negative shift to each particle's position in the chain axis, and measure the root-mean-square of the disorder as a percentage of $\Lambda=|\beta-1|$. When the disorder is $50 \%$ the system is within one standard deviation of the topological phase transition, where the particles are equally spaced.

In Figure 6a, a random choice of disorder is scaled smoothly for different choices of $\beta$, causing the bulk modes (blue) to enter and eventually close the gap at around 50\%. These bulk modes also become localized, ${ }^{76}$ with example mode profiles in Figure $6 \mathrm{~b}$. The edge modes (yellow) separate in energy until they are lost in the bulk, but survive for high levels (sometimes greater than $20 \%$ ) of disorder, especially for larger $|\beta-1|$. Figure $6 \mathrm{c}$ shows the mode profiles of the two edge modes for two joined chains with $20 \%$ disorder and opposite Zak phases, which illustrates the continued existence of the edge modes in disordered systems. These disorder-protected edge modes act as plasmonic hotspots, which can be positioned anywhere at the interface of two chains with opposite Zak phase.

Complex Bands. In any system featuring losses or retardation, one of the wavevector or frequency must be complex. $^{77}$ This is typically chosen depending on the physics of the studied system. Here we discuss the importance of this with regards to a plasmonic topological insulator.

For non-Hermitian solid state topological insulators the eigenvalues are complex and the Bloch wavevector has real components, making the choice of real $k_{x}$ and complex frequency natural for comparison. Figure $7 \mathrm{a}$ shows the real and imaginary parts of the Bloch band structure (red and green lines) in the case where there is a crossing in the real frequency axis, and the gap still has an imaginary part. We can visualize these bands as curves embedded in three dimensions as in Figure $7 \mathrm{~b}$. It is necessary to take into account both the real and 
Longitudinal $d=330 \mathrm{~nm}, a=20 \mathrm{~nm}, \beta=1.1$

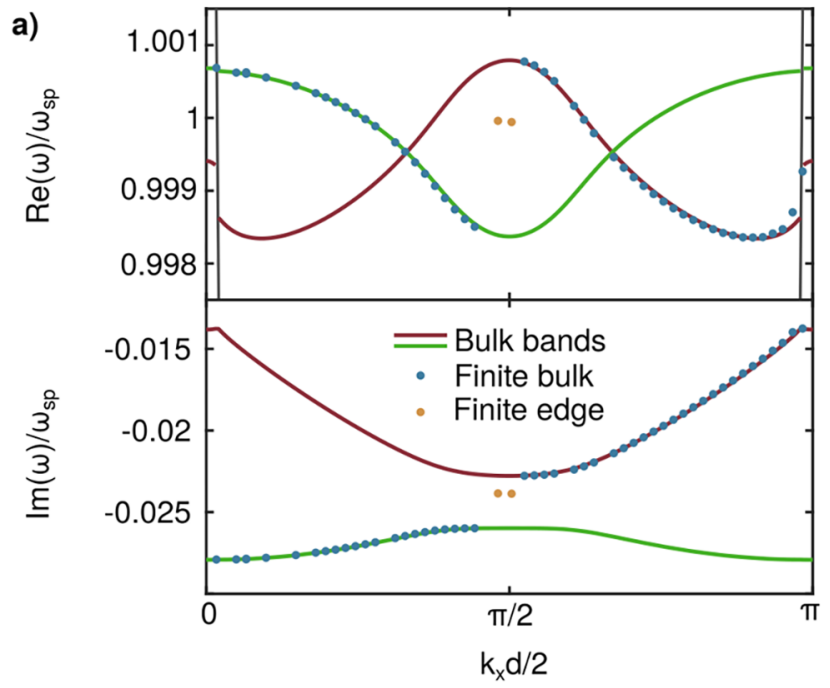

b)

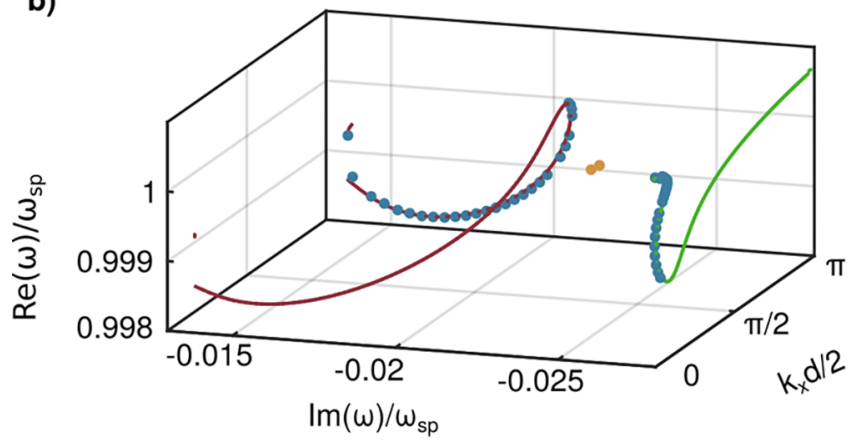

Figure 7. (a) Real and imaginary parts of the band structure (red and green lines) for $d=300 \mathrm{~nm}, a=20 \mathrm{~nm}, \beta=1.1$, overlaid with modes for a finite 60 particle chain of the same geometry (blue and yellow dots). The two bulk bands are colored differently for clarity. (b) 3D representation of the above two projections, demonstrating that the curve is embedded in a $3 \mathrm{D}$ space.

the imaginary parts of the frequency when considering the topological nature of a non-Hermitian system. ${ }^{78}$ Here the complex gap allows for the unambiguous identification of each band, which are labeled with different colors for clarity. This then permits the calculation of Zak phases. For a finite chain, we still find bulk modes (blue dots) which follow the bands and edge modes (yellow dots) which sit in the complex valued gap.

All of the retarded and radiative band structures presented in this work have imaginary components to the frequency, whose inverse is equal to the lifetime of the mode. Lifetimes of edge modes are comparable to those of the bulk modes and plasmons localized to a single particle; for example, the edge modes in Figure 7 have a lifetime on the order of $10 \mathrm{fs}$. The presence of these imaginary parts suggest that it may be necessary to use an evanescent wave to excite the modes.

\section{CONCLUSION}

We have presented a detailed study of the $1 \mathrm{D}$ topological plasmonic chain beyond the quasistatic limit. We have discussed how appropriately modeling the interaction between the plasmonic nanoparticles by including the effects of retardation and radiative damping, as well as losses in the metal, leads to fundamental differences with its original electronic analogue, the SSH model. In particular, the plasmonic chain has a non-Hermitian Hamiltonian with longrange hopping which breaks chiral symmetry in a "trivial" way. This implies that, because the system has the same eigenvectors as a chirally symmetric counterpart, it is still a topologically nontrivial system that supports edge modes at interfaces between topological phases.

While in the QS limit most of the behavior of the SSH model is recovered, specifically real frequency band gaps opening both for transverse and longitudinal modes and edge modes confined to a single sublattice in the topological phase, we have shown that as the size of the particles and their separation increases to more experimentally appropriate scales a richer phenomenology appears. In particular, the bulk band structures deviate strongly from the QS prediction, and a band gap opening for the dimerized chain does not always appear in the real frequency axis. However, in these cases, there is still a gap in the imaginary part of the frequency so that it is possible to calculate topological invariants and define topological phases. We have calculated the Zak phase and discovered remarkable retardation-induced topological phase changes for the transverse modes, which are due specifically to the long-range term in the Green's function.

We confirmed that the edge states survive positional disorder in the axis of the chain to a great extent. This hints at potential uses for the $1 \mathrm{D}$ topological plasmonic chain in plasmonic systems, which could take advantage of this robustness against fabrication imperfections to design plasmonic hotspots.

Finally, we discussed the implications of non-Hermiticity and complex valued frequencies which arise due to loss and retardation. Although the real and imaginary parts of the frequency $\omega$ have different physical interpretations, they are equally important in determining the topology of the system.

\section{ASSOCIATED CONTENT}

\section{Supporting Information}

The Supporting Information is available free of charge on the ACS Publications website at DOI: 10.1021/acsphotonics.8b00117.

Additional details on the Lerch transcendent form and trivial chiral symmetry breaking, as well as commercial software band structures for comparison with those in the main text $(\mathrm{PDF})$.

\section{AUTHOR INFORMATION}

\section{Corresponding Author}

*E-mail: s.pocock15@imperial.ac.uk.

\section{ORCID}

Simon R. Pocock: 0000-0002-5954-2205

Xiaofei Xiao: 0000-0002-7053-2833

\section{Author Contributions}

S.R.P. performed the research and wrote the manuscript. X.X. and P.A.H. performed band structure calculations with FDTD and FEM solvers for Supporting Information. P.A.H. and V.G. supervised the research and provided guidance. V.G. designed the project.

\section{Notes}

The authors declare no competing financial interest.

${ }^{\S}$ Group web page: www.gianninilab.com 


\section{ACKNOWLEDGMENTS}

S.R.P. thanks S. Lieu for many enlightening conversations about topological insulators, and acknowledges funding from EPSRC. X.X. acknowledges support from a Lee Family Scholarship. P.A.H. acknowledges funding from a Marie Skłodowska-Curie Fellowship.

\section{REFERENCES}

(1) Maier, S. A. Plasmonics: Fundamentals and Applications, 1st ed.; Springer: U.S., 2007.

(2) Giannini, V.; Fernández-Domínguez, A. I.; Heck, S. C.; Maier, S. A. Plasmonic Nanoantennas: Fundamentals and Their Use in Controlling the Radiative Properties of Nanoemitters. Chem. Rev. 2011, 111, 3888-3912.

(3) Zou, S.; Schatz, G. C. Theoretical studies of plasmon resonances in one-dimensional nanoparticle chains: narrow lineshapes with tunable widths. Nanotechnology 2006, 17, 2813-2820.

(4) de Abajo, F. J. G. Colloquium: Light scattering by particle and hole arrays. Rev. Mod. Phys. 2007, 79, 1267-1290.

(5) Auguié, B.; Barnes, W. L. Collective Resonances in Gold Nanoparticle Arrays. Phys. Rev. Lett. 2008, 101, 143902.

(6) Barrow, S. J.; Rossouw, D.; Funston, A. M.; Botton, G. A.; Mulvaney, P. Mapping Bright and Dark Modes in Gold Nanoparticle Chains using Electron Energy Loss Spectroscopy. Nano Lett. 2014, 14, 3799-3808.

(7) Zhou, W.; Dridi, M.; Suh, J. Y.; Kim, C. H.; Co, D. T.; Wasielewski, M. R.; Schatz, G. C.; Odom, T. W. Lasing action in strongly coupled plasmonic nanocavity arrays. Nat. Nanotechnol. 2013, $8,506-511$.

(8) Hakala, T. K.; Rekola, H. T.; Väkeväinen, A. I.; Martikainen, J.-P.; Nečada, M.; Moilanen, A. J.; Törmä, P. Lasing in dark and bright modes of a finite-sized plasmonic lattice. Nat. Commun. 2017, 8, 13687.

(9) Giannini, V.; Vecchi, G.; Gómez Rivas, J. Lighting Up Multipolar Surface Plasmon Polaritons by Collective Resonances in Arrays of Nanoantennas. Phys. Rev. Lett. 2010, 105, 266801.

(10) Todisco, F.; Esposito, M.; Panaro, S.; de Giorgi, M.; Dominici, L.; Ballarini, D.; Fernández-Domínguez, A. I.; Tasco, V.; Cuscunà, M.; Passaseo, A.; Ciracì, C.; Gigli, G.; Sanvitto, D. Toward Cavity Quantum Electrodynamics with Hybrid Photon Gap-Plasmon States. ACS Nano 2016, 10, 11360-11368.

(11) Humphrey, A. D.; Meinzer, N.; Starkey, T. A.; Barnes, W. L. Surface Lattice Resonances in Plasmonic Arrays of Asymmetric Disc Dimers. ACS Photonics 2016, 3, 634-639.

(12) Han, D.; Lai, Y.; Zi, J.; Zhang, Z.-Q.; Chan, C. T. Dirac Spectra and Edge States in Honeycomb Plasmonic Lattices. Phys. Rev. Lett. 2009, 102, 123904.

(13) Khanikaev, A. B.; Mousavi, S. H.; Tse, W.-K.; Kargarian, M.; MacDonald, A. H.; Shvets, G. Photonic Topological Insulators. Nat. Mater. 2013, 12, 233-239.

(14) Weick, G.; Woollacott, C.; Barnes, W. L.; Hess, O.; Mariani, E. Dirac-like Plasmons in Honeycomb Lattices of Metallic Nanoparticles. Phys. Rev. Lett. 2013, 110, 106801.

(15) Poddubny, A.; Miroshnichenko, A.; Slobozhanyuk, A.; Kivshar, Y. Topological Majorana States in Zigzag Chains of Plasmonic Nanoparticles. ACS Photonics 2014, 1, 101-105.

(16) Sinev, I. S.; Mukhin, I. S.; Slobozhanyuk, A. P.; Poddubny, A. N.; Miroshnichenko, A. E.; Samusev, A. K.; Kivshar, Y. S. Mapping plasmonic topological states at the nanoscale. Nanoscale 2015, 7, 11904-11908.

(17) Wang, L.; Zhang, R. Y.; Xiao, M.; Han, D.; Chan, C. T.; Wen, $\mathrm{W}$. The existence of topological edge states in honeycomb plasmonic lattices. New J. Phys. 2016, 18, 103029.

(18) Jin, D.; Christensen, T.; Soljačić, M.; Fang, N. X.; Lu, L.; Zhang, X. Infrared Topological Plasmons in Graphene. Phys. Rev. Lett. 2017, $118,245301$.
(19) Bleckmann, F.; Cherpakova, Z.; Linden, S.; Alberti, A. Spectral imaging of topological edge states in plasmonic waveguide arrays. Phys. Rev. B: Condens. Matter Mater. Phys. 2017, 96, 045417.

(20) Zhang, W.; Chen, X.; Ye, F. Plasmonic topological insulators for topological nanophotonics. Opt. Lett. 2017, 42, 4063-4066.

(21) Qiu, P.; Liang, R.; Qiu, W.; Chen, H.; Ren, J.; Lin, Z.; Wang, J.X.; Kan, Q.; Pan, J.-Q. Topologically protected edge states in graphene plasmonic crystals. Opt. Express 2017, 25, 22587-22594.

(22) Yang, B.; Guo, Q.; Tremain, B.; Barr, L. E.; Gao, W.; Liu, H.; Béri, B.; Xiang, Y.; Fan, D.; Hibbins, A. P.; Zhang, S. Direct observation of topological surface-state arcs in photonic metamaterials. Nat. Commun. 2017, 8, 97.

(23) Saba, M.; Hamm, J. M.; Baumberg, J. J.; Hess, O. Group Theoretical Route to Deterministic Weyl Points in Chiral Photonic Lattices. Phys. Rev. Lett. 2017, 119, 227401.

(24) Bliokh, K. Y.; Smirnova, D.; Nori, F. Quantum spin Hall effect of light. Science 2015, 348, 1448-1451.

(25) St-Jean, P.; Goblot, V.; Galopin, E.; Lemaitre, A.; Ozawa, T.; Gratiet, L. L.; Sagnes, I.; Bloch, J.; Amo, A. Lasing in topological edge states of a one-dimensional lattice. Nat. Photonics 2017, 11, 651-656.

(26) Yang, A.; Hryn, A. J.; Bourgeois, M. R.; Lee, W.-K.; Hu, J.; Schatz, G. C.; Odom, T. W. Programmable and reversible plasmon mode engineering. Proc. Natl. Acad. Sci. U. S. A. 2016, 113, 1420114206.

(27) Mostafazadeh, A. A new class of adiabatic cyclic states and geometric phases for non-Hermitian Hamiltonians. Phys. Lett. A 1999, 264, 11-17.

(28) Malzard, S.; Poli, C.; Schomerus, H. Topologically Protected Defect States in Open Photonic Systems with Non-Hermitian ChargeConjugation and Parity-Time Symmetry. Phys. Rev. Lett. 2015, 115, 200402.

(29) Ling, C. W.; Choi, K. H.; Mok, T. C.; Zhang, Z.-Q.; Fung, K. H. Anomalous Light Scattering by Topological PT-Symmetric Particle Arrays. Sci. Rep. 2016, 6, 38049.

(30) Ke, S.; Wang, B.; Long, H.; Wang, K.; Lu, P. Topological edge modes in non-Hermitian plasmonic waveguide arrays. Opt. Express 2017, 25, 11132-11143.

(31) Wiemann, S.; Kremer, M.; Plotnik, Y.; Lumer, Y.; Nolte, S.; Makris, K. G.; Segev, M.; Rechtsman, M. C.; Szameit, A. Topological protected bound states in photonic parity-time-symmetric crystals. Nat. Mater. 2017, 16, 433-438.

(32) Lieu, S. Topological phases in the non-Hermitian Su-SchriefferHeeger model. Phys. Rev. B: Condens. Matter Mater. Phys. 2018, 97, 045106.

(33) Lu, L.; Joannopoulos, J. D.; Soljačić, M. Topological photonics. Nat. Photonics 2014, 8, 821-829.

(34) Lu, L.; Joannopoulos, J. D.; Soljačić, M. Topological states in photonic systems. Nat. Phys. 2016, 12, 626-629.

(35) Sun, X.-C.; He, C.; Liu, X.-P.; Lu, M.-H.; Zhu, S.-N.; Chen, Y.-F. Two-dimensional topological photonic systems. Prog. Quantum Electron. 2017, 55, 52-73.

(36) Siroki, G.; Huidobro, P. A.; Giannini, V. Topological Photonics: From Crystals to Particles. Phys. Rev. B: Condens. Matter Mater. Phys. 2017, 96, 041408.

(37) Su, W. P.; Schrieffer, J. R.; Heeger, A. J. Solitons in Polyacetylene. Phys. Rev. Lett. 1979, 42, 1698-1701.

(38) Asbóth, J. K.; Oroszlány, L.; Pályi, A. A Short Course on Topological Insulators; Springer International Publishing: Switzerland, 2016.

(39) Slobozhanyuk, A. P.; Poddubny, A. N.; Miroshnichenko, A. E.; Belov, P. A.; Kivshar, Y. S. Subwavelength Topological Edge States in Optically Resonant Dielectric Structures. Phys. Rev. Lett. 2015, 114, 123901.

(40) Solnyshkov, D. D.; Nalitov, A. V.; Malpuech, G. Kibble-Zurek Mechanism in Topologically Nontrivial Zigzag Chains of Polariton Micropillars. Phys. Rev. Lett. 2016, 116, 046402.

(41) Ling, C. W.; Xiao, M.; Chan, C. T.; Yu, S. F.; Fung, K. H. Topological edge plasmon modes between diatomic chains of plasmonic nanoparticles. Opt. Express 2015, 23, 2021-2031. 
(42) Downing, C. A.; Weick, G. Topological collective plasmons in bipartite chains of metallic nanoparticles. Phys. Rev. B: Condens. Matter Mater. Phys. 2017, 95, 125426.

(43) Gómez, D. E.; Hwang, Y.; Lin, J.; Davis, T. J.; Roberts, A. Plasmonic Edge States: An Electrostatic Eigenmode Description. ACS Photonics 2017, 4, 1607-1614.

(44) Weber, W. H.; Ford, G. W. Propagation of optical excitations by dipolar interacitons in metal nanoparticle chains. Phys. Rev. B: Condens. Matter Mater. Phys. 2004, 70, 125429.

(45) Simovski, C. R.; Viitanen, A. J.; Tretyakov, S. A. Resonator mode in chains of silver spheres and its possible application. Phys. Rev. E 2005, 72, 066606.

(46) Koenderink, A. F.; Polman, A. Complex response and polaritonlike dispersion splitting in periodic metal nanoparticle chains. Phys. Rev. B: Condens. Matter Mater. Phys. 2006, 74, 033402.

(47) Downing, C. A.; Mariani, E.; Weick, G. Retardation effects on the dispersion and propagation of plasmons in metallic nanoparticle chains. J. Phys.: Condens. Matter 2018, 30, 025301.

(48) Compaijen, P. J.; Malyshev, V. A.; Knoester, J. Time-dependent transport of a localized surface plasmon through a linear array of metal nanoparticles: Precursor and normal mode contributions. Phys. Rev. B: Condens. Matter Mater. Phys. 2018, 97, 085428.

(49) Zhu, X.; Vannahme, C.; Højlund-Nielsen, E.; Mortensen, N. A.; Kristensen, A. Plasmonic colour laser printing. Nat. Nanotechnol. 2015, 11, 325-329.

(50) Ross, M. B.; Mirkin, C. A.; Schatz, G. C. Optical Properties of One-, Two-, and Three-Dimensional Arrays of Plasmonic Nanostructures. J. Phys. Chem. C 2016, 120, 816-830.

(51) Genevet, P.; Capasso, F.; Aieta, F.; Khorasaninejad, M.; Devlin, R. Recent advances in planar optics: from plasmonic to dielectric metasurfaces. Optica 2017, 4, 139-152.

(52) Downing, C. A.; Weick, G. Topological plasmons in dimerized chains of nanoparticles: robustness against long-range quasistatic interactions and retardation effects. ArXiv e-prints 2018, arXiv: 1803.08872 .

(53) Li, L.; Xu, Z.; Chen, S. Topological phases of generlized SuSchrieffer-Heeger models. Phys. Rev. B: Condens. Matter Mater. Phys. 2014, 89, 085111.

(54) Zhu, B.; Lü, R.; Chen, S. PT symmetry in the non-Hermitian Su-Schrieffer-Heeger model with complex boundary potentials. Phys. Rev. A: At., Mol., Opt. Phys. 2014, 89, 062102.

(55) Chen, B.-H.; Chiou, D.-W. An elementary proof of bulkboundary correspondence in the generalized Su-Schrieffer-Heeger model. ArXiv e-prints 2017, arXiv:1705.06913.

(56) Pérez-González, B.; Bello, M.; Gómez-León, Á.; Platero, G. SSH model with long-range hoppings: topology, driving and disorder. ArXiv e-prints 2018, arXiv: 1802.03973.

(57) Park, S. Y.; Stroud, D. Surface-plasmon dispersion relations in chains of metallic nanoparticles: An exact quasistatic calculation. Phys. Rev. B: Condens. Matter Mater. Phys. 2004, 69, 125418.

(58) Novotny, L.; Hecht, B. Principles of Nano-Optics; Cambridge University Press: Cambridge, 2006.

(59) Vial, A.; Grimault, A.-S.; Macias, D.; Barchiesi, D.; de la Chapelle, M. L. Improved analytical fit of gold dispersion: Application to the modeling of extinction spectra with a finite-difference timedomain method. Phys. Rev. B: Condens. Matter Mater. Phys. 2005, 71, 085416.

(60) Compaijen, P. J.; Malyshev, V. A.; Knoester, J. Surface-mediated light transmission in metal nanoparticle chains. Phys. Rev. B: Condens. Matter Mater. Phys. 2013, 87, 205437.

(61) Compaijen, P. J.; Malyshev, V. A.; Knoester, J. Engineering plasmon dispersion relations: hybrid nanoparticle chain -substrate plasmon polaritons. Opt. Express 2015, 23, 2280-2292.

(62) Fitzgerald, J. M.; Narang, P.; Craster, R. V.; Maier, S. A.; Giannini, V. Quantum Plasmonics. Proc. IEEE 2016, 104, 2307-2322.

(63) Alù, A.; Engheta, N. Theory of linear chains of metamaterial/ plasmonic particles as subdiffraction optical nanotransmission lines. Phys. Rev. B: Condens. Matter Mater. Phys. 2006, 74, 205436.
(64) Alù, A.; Engheta, N. Guided propagation along quadrupolar chains of plasmonic nanoparticles. Phys. Rev. B: Condens. Matter Mater. Phys. 2009, 79, 235412.

(65) Conforti, M.; Guasoni, M. Dispersive properties of linear chains of lossy metal nanoparticles. J. Opt. Soc. Am. B 2010, 27, 1576-1582.

(66) Udagedara, I. B.; Rukhlenko, I. D.; Premaratne, M. Surface plasmon-polariton propagation in piecewise linear chains of composite nanospheres: The role of optical gain and chain layout. Opt. Express 2011, 19, 19973-19986.

(67) Rolly, B.; Bonod, N.; Stout, B. Dispersion relations in metal nanoparticle chains: necessity of the multipole approach. J. Opt. Soc. Am. B 2012, 29, 1012-1019.

(68) Hadad, Y.; Mazor, Y.; Steinberg, B. Z. Green's function theory for one-way particle chains. Phys. Rev. B: Condens. Matter Mater. Phys. 2013, 87, 035130.

(69) Jacak, W. A. Exact solution for velocity of plasmon-polariton in metallic nano-chain. Opt. Express 2014, 22, 18958-18965.

(70) Petrov, M. Disorder-induced Purcell enhancement in nanoparticle chains. Phys. Rev. A: At., Mol., Opt. Phys. 2015, 91, 023821.

(71) Ordonez-Miranda, J.; Tranchant, L.; Gluchko, S.; Volz, S. Energy transport of surface phonon polaritons propagating along a chain of spheroidal nanoparticles. Phys. Rev. B: Condens. Matter Mater. Phys. 2015, 92, 115409.

(72) Zak, J. Berry's phase for energy bands in solids. Phys. Rev. Lett. 1989, 62, 2747-2750.

(73) Delplace, P.; Ullmo, D.; Montambaux, G. Zak phase and the existence of edge states in graphene. Phys. Rev. B: Condens. Matter Mater. Phys. 2011, 84, 195452 .

(74) van Miert, G.; Ortix, C.; Smith, C. M. Topological origin of edge states in two-dimensional inversion-symmetric insulators and semimetals. 2D Mater. 2017, 4, 015023.

(75) Xiao, M.; Zhang, Z. Q.; Chan, C. T. Surface Impedance and Bulk Band Geometric Phases in One-Dimensional Systems. Phys. Rev. $X$ 2014, 4, 021017.

(76) Markel, V. A.; Sarychev, A. K. Propagation of surface plasmons in ordered and disordered chains of metal nanospheres. Phys. Rev. B: Condens. Matter Mater. Phys. 2007, 75, 085426.

(77) Le Gall, J.; Olivier, M.; Greffet, J.-J. Experimental and theoretical study of reflection and coherent thermal emissionby a $\mathrm{SiC}$ grating supporting a surface-phonon polariton. Phys. Rev. B: Condens. Matter Mater. Phys. 1997, 55, 10105-10114.

(78) Shen, H.; Zhen, B.; Fu, L. Topological Band Theory for NonHermitian Hamiltonians. Phys. Rev. Lett. 2018, 120, 146402. 